\title{
Design multi-stopband laminate acoustic metamaterials for structural-acoustic coupled system
}

\author{
$\mathrm{XXiao}{ }^{1}, \mathrm{ZC} \mathrm{He}^{1,3^{*}},{\text { Eric } \mathrm{Li}^{2 *}, \mathrm{AG} \text { Cheng }}^{1}$ \\ ${ }^{1}$ State Key Laboratory of Advanced Design and Manufacturing for Vehicle Body, Hunan University, Changsha, \\ 410082 P. R. China \\ ${ }^{2}$ School of Science, Engineering \& Design, Teesside University, Middlesbrough, UK ${ }^{3}$ The State Key \\ ${ }^{3}$ Laboratory of Fluid Power and Mechatronic Systems, Zhejiang University, Hangzhou,
} 310027, China

\begin{abstract}
This paper designs the laminate acoustic metamaterials with multi-stopband for structuralacoustic coupled system. The laminate acoustic metamaterials are composed of carbon-fiberreinforced polymer (CFRP) and a periodic array of two degrees of freedom (2-DOF) massspring-damper subsystems attached to the laminate. According to the dispersion analysis, two stopbands are observed around the absorbers' resonant frequency. Based on the finite element modeling, the multi-stopband behavior has been confirmed. In addition, the effects of damping of vibration absorbers are discussed in this work. By adding the appropriate damping to the vibration absorbers, the two stop bands can be combined into a wider stopband. Subsequently, the analyses of multi-stopband laminate acoustic metamaterials in the structural-acoustic coupled system are performed. The excellent performance of multi-stopband laminate acoustic metamaterials has been applied to the front panel of vehicle, and the noise of passenger compartment cavity is reduced significantly.
\end{abstract}

Key words: laminate acoustic metamaterials; stop-band; structural-acoustic coupled system; local resonance

*Corresponding author: ZC He, Eric Li

Email address: hezhicheng815@hnu.edu.cn (ZC He); ericsg2012@gmail.com (Eric Li) 


\section{Introduction}

In the late 1960s, the concept of metamaterials was first proposed by Veselago [1] and used in the field of electromagnetic waves. Veselago has theoretically proved that materials with negative constitutive parameters can exist and be manufactured. Due to the mathematical similarity between the acoustic and electromagnetic waves, acoustic bandgap materials known as phononic crystals were proposed recently [2-10]. Phononic crystals are usually constructed of two materials with distinctive elastic properties, and the periodic arrangement of the two materials causes the destructive Bragg scattering of the acoustic waves propagating within the phononic crystal, thereby forming a stopband or bandgap in a particular frequency range. However, the bandgap frequency is fundamentally dependent on the geometry of basic scatterer. More specifically, the wavelength of the acoustic wave corresponding to this type of bandgap is the same order of magnitude of the lattice constant.

Due to the limitations of Bragg type phononic crystals in low frequency applications, Liu et al. took the lead in applying the concept of local resonance to the phononic crystals [11]. This type of phononic crystals is also known as local resonance acoustic metamaterials (LRAMs) [12]. The LRAMs are composite materials in which the basic scatterer is further designed as microlocal resonator and the bandgap can be obtained two orders of magnitude lower than that of the Bragg gaps. The in-depth studies found that the local resonance can produce exotic acoustic material characteristics such as negative effective mass density [11, 13-17], negative effective bulk modulus $[18]$ or shear modulus $[19,20]$ as well as simultaneously negative modulus and mass [21-24]. Some new concepts that exploit these special properties have emerged, including superlensing [25-27], cloaking [28-32], subwavelength imaging [33] and artificial acoustic black hole $[24,34]$. 
Recently, acoustic metamaterials with engineering structures (such as bars, beams and plates) have gained widespread attention. The pioneering idea of local resonance was applied in engineering structures by Wang et al [35]. A simple quasi-one-dimensional mechanical analog model was introduced to investigate the physical insight of the locally resonant mechanism. In 2006, Wang et al. proposed a basic model of locally resonant bars [36]. The propagation of longitudinal elastic waves in locally resonant bars was studied. In both theoretical and experimental results, the sub-frequency locally resonant band gap with highly asymmetric attenuation was observed and two results were in great agreement. Yu et al. have studied the flexural vibration in Timoshenko beams with periodical local resonators theoretically and experimentally [37]. Then the model was improved with double mass-spring subsystem integrated to the Euler-Bernoulli beams [38]. The measurements showed that an attenuation of more than $20 \mathrm{~dB}$ occurred over the frequency range of the bandgaps. The existence of the low frequency bandgaps in beams provides a method for flexural vibration control of beams. Oudich et al. theoretically demonstrated the waveguiding of Lamb waves in a two-dimensional locally resonant plate $[39,40]$. They mainly focused on the formation of the complete bandgap of Lamb waves propagating in the locally resonant plate and the influence of the parameters, but the experimental verification was not involved. In 2011, Oudich et al. provided experimental evidence for the existence of locally resonant acoustic band gaps in two-dimensional plates [41]. The locally resonant acoustic bandgap at about $2.2 \mathrm{kHz}$ was observed. In addition, the potential of locally resonant thin plates with a periodic array of resonators for the application of low frequency vibration suppression has been investigated[42-44].

In short, the dynamic vibration absorber as a substitute for the mass-spring subsystem can be combined with bars, beams, plates and other engineering structures to form the locally 
resonant structures[45]. The purpose of vibration and noise reduction would be achieved by utilizing the bandgap characteristics of periodic structures. It is found from above structures that the stiffness and mass are two factors that affect the actual attenuation of the locally resonant bandgap. Review of the existing locally resonant structures [35-41, 46, 47], it can be seen that the mass of the dynamic vibration absorber is much greater than that of the substrate, which is very unfavorable in engineering applications. Therefore, it is necessary to consider not only the weight of the structure but also the low-frequency range of the bandgap. At the same time, due to the limitations of the oscillator mass, a smaller stiffness of the oscillator is preferred in order to obtain the low-frequency bandgap. Hence the equivalent stiffness of the substrate should be appropriately reduced, which will lead to lower overall system stiffness. Thus, it would be a great idea to integrate the composite materials like carbon-fiber-reinforced polymer (CFRP) [48], which have high ratio of strength to weight, into acoustic metamaterials to control the propagation of low-frequency sonic waves. Composite materials have recently been widely used in the automotive, aerospace and other fields due to its light weight and good performance [49]. The material property of the composites can be engineered according to the application requirements [48, 50-52]. However, some undesirable behaviors like extensional-shear coupling and bending-twist coupling may exist in unsymmetrical laminate, which can cause performance degradation in the application of composite materials [53]. Therefore, the symmetrical laminate widely used in engineering is applied to design acoustic metamaterials to avoid these unsatisfactory behaviors.

In addition, with the increasing demand for Noise Vibration and Harshness (NVH) performance, noise control of the closed cavity such as aircraft cabins and passenger compartments has become an urgent problem for engineers. It has been found that the interior 
noise is affected significantly by the structural vibration, acoustic domain and coupled interface.

The structural vibration produces noise, and noise in turn reacts to the structure as a result of the sound pressure. The acoustic analysis of structural-acoustic coupled system based on finite element method (FEM) can predict the vibration and noise in the early design stage[54, 55]. It can be widely used in the design of aircraft and vehicle for vibration and noise control.

This work aims to design multi-stopband laminate acoustic metamaterials with the superior strength to weight ratio for broadband vibration absorption and noise reduction in the structuralacoustic coupled system. The multi-stopband laminate acoustic metamaterials consist of orthotropic laminates and multi-frequency mass-spring-damper, which show the extraordinary performance for noise and vibration mitigation in the structural-acoustic coupling system. The paper is structured as follows. Firstly, in Section 2, the dynamic equilibrium equation of the structural-acoustic coupled system is derived in detail. The dispersion analysis of the multistopband behavior of laminate acoustic metamaterials is conducted in Section 3. Afterwards, the multi-stopband of laminate acoustic metamaterials is verified by frequency response analysis. In addition, the working mechanism of the bandgaps is revealed based on the concept of conventional dynamic vibration absorbers. The influences of the absorbers' resonant frequencies and damping ratios of the multi-frequency vibration absorbers as well as the laminate's mode shapes are investigated. In Section 4, two numerical models are conducted to demonstrate the excellent performance of the multi-stopband laminate for interior noise and vibration reduction. Finally, the results as well as the conclusions drawn from the paper are given.

\section{Basic theory of laminate acoustic metamaterials for structural-acoustic coupled system}

The structural-acoustic coupled system with laminate acoustic metamaterials is shown in Fig. 1. The system consists of three parts: the structure domain $\Omega_{s}$, the acoustic domain $\Omega_{f}$, 
and the coupled domain $\partial \Omega_{s f}$ [56-60]. The external and natural boundaries are denoted as $\Gamma_{u}$ and $\Gamma_{t}$ respectively. The acoustic boundary is expressed as $\Gamma_{f}$. The dynamic equation of each part is investigated using FEM.

\subsection{FEM model for the structure domain}

According to the Kirchhoff Plate theory, the stiffness matrix of the ply on the global coordinate can be expressed as follows[45]:

$$
\begin{aligned}
& \bar{K}_{11}=\cos ^{4} \theta K_{11}+\sin ^{4} \theta K_{22}+2 \cos ^{2} \theta \sin ^{2} \theta\left(K_{12}+2 K_{44}\right) \\
& \bar{K}_{22}=\sin ^{4} \theta K_{11}+\cos ^{4} \theta K_{22}+2 \cos ^{2} \theta \sin ^{2} \theta\left(K_{12}+2 K_{44}\right) \\
& \bar{K}_{44}=\left(K_{11}+K_{22}-2 K_{12}\right) \cos ^{2} \theta \sin ^{2} \theta+\left(\cos ^{2} \theta-\sin ^{2} \theta\right) K_{44} \\
& \bar{K}_{12}=\bar{K}_{21}=\left(\cos ^{4} \theta+\sin ^{4} \theta\right) K_{12}+\left(K_{11}+K_{22}-4 K_{44}\right) \cos ^{2} \theta \sin ^{2} \theta \\
& \bar{K}_{14}=\bar{K}_{41}=\left[\cos ^{2} \theta\left(K_{11}-K_{12}-2 K_{44}\right)+\sin ^{2} \theta\left(K_{12}-K_{22}+2 K_{44}\right)\right] \cos \theta \sin \theta \\
& \bar{K}_{24}=\bar{K}_{42}=\left[\cos ^{2} \theta\left(K_{12}-K_{22}+2 K_{44}\right)+\sin ^{2} \theta\left(K_{11}-K_{12}-2 K_{44}\right)\right] \cos \theta \sin \theta
\end{aligned}
$$

where $\theta$ represents the angle between the principal material coordinate system and global coordinate system, and $[\mathbf{K}]$ is the stiffness matrix on the principal material coordinate.

The discrete form of the dynamic equilibrium equation in the non-damping structuralacoustic system is[58]

$$
\mathbf{M}_{s} \ddot{u}_{s}+\mathbf{K}_{s} u_{s}=\mathbf{F}_{e}+\mathbf{F}_{s}
$$

where $\mathbf{M}_{s}$ and $\mathbf{K}_{s}$ represent the structural mass and stiffness matrices, respectively, $\mathbf{F}_{e}$ and $\mathbf{F}_{s}$ stand for the external body load vector applied to the structure and the surface load vector arising from the acoustic domain.

$\mathbf{F}_{s}$ can be denoted as: 


$$
\mathbf{F}_{s}=-\int_{\partial \Omega_{s f}} \mathbf{N}_{s}^{\mathrm{T}} \tau d \Gamma
$$

where $\mathbf{N}_{s}$ represents the Lagrange shape function of the structure element, and $\tau$ represents the surface traction force arising from the acoustic domain.

\subsection{FEM model for the acoustic domain}

The radiated steady-state acoustic pressure $p$ of the structure under a harmonic excitation is governed by Helmholtz equation [61, 62]:

$$
\nabla^{2}\left(p e^{i \omega t}\right)-\frac{1}{c^{2}} \frac{\partial^{2}\left(p e^{i \omega t}\right)}{\partial t^{2}}=0
$$

where $\nabla^{2}$ is the Laplace operator, $t$ is the time, $c$ is the sound speed, and $\omega$ is the angular frequency.

The Garlerkin weak form of the acoustic dynamic equation can be expressed regardless of the damping of acoustic domain:

$$
\int_{\Omega_{f}}\left(\delta \nabla p \cdot \nabla p+\frac{1}{c^{2}} \delta p \cdot p\right) d \Omega-\rho_{f} \int_{\partial \Omega_{s f}} \delta p \cdot \ddot{u}_{f} d \Gamma-\int_{\Omega_{f}} \delta p \cdot \frac{\partial q_{f}}{\partial t} d \Omega=0
$$

where $\ddot{u}_{f}$ is the normal acceleration of the acoustic fluid attached to the structure, $\rho_{f}$ is the density of acoustic fluid domain, and $q_{f}$ is the external load of unit volume.

By applying FEM, the acoustic domain is discretized into a number of isoparametric hexahedral elements, and the acoustic pressure $p$ can be approximated as

$$
p=\mathrm{N}_{f} \mathbf{p}
$$

where $\mathrm{N}_{f}$ is the Lagrange shape function of isoparametric hexahedral elements, and $\mathbf{p}$ is the pressure vector in the node of isoparametric hexahedral element. 
Substituting Eq. (6) into (5), the acoustic discrete dynamic equilibrium equation without the damping can be established:

$$
\mathbf{K}_{f} \mathbf{p}+\mathbf{M}_{f} \ddot{\mathbf{p}}=\mathbf{F}_{f}+\mathbf{F}_{q}
$$

where $\mathbf{M}_{f}$ and $\mathbf{K}_{f}$ represent the acoustic mass matrix and stiffness matrix respectively, $\mathbf{F}_{q}$ and $\mathbf{F}_{f}$ stand for the external load vector applied to the acoustic and the surface load vector arising from the structure domain. They can be obtained as follows:

$$
\begin{gathered}
\mathbf{K}_{f}=\int_{\Omega_{f}}\left(\nabla \mathbf{N}_{f}\right)^{\mathrm{T}}\left(\nabla \mathbf{N}_{f}\right) d \Omega \\
\mathbf{M}_{f}=\frac{1}{c^{2}} \int_{\Omega_{f}} \mathbf{N}_{f}{ }^{\mathrm{T}} \mathbf{N}_{f} d \Omega \\
\mathbf{F}_{q}=\int_{\Omega_{f}} \mathbf{N}_{f}{ }^{\mathrm{T}} \frac{\partial q_{f}}{\partial t} d \Omega \\
\mathbf{F}_{f}=\rho \int_{\Omega_{s f}} \mathbf{N}_{f}{ }^{\mathrm{T}} \ddot{u}_{f} d \Gamma
\end{gathered}
$$

\subsection{FEM model for the coupled domain}

The coupled interface $\partial \Omega_{s f}$ in the structure-acoustic system should satisfy the displacement and pressure continuity conditions.

$$
\mathrm{u}_{s} \mathbf{n}=\mathrm{u}_{f} \mathbf{n},\left.\tau\right|_{n}=-p
$$

where $\mathbf{n}$ is the normal vector of the coupled interface, $u_{s}$ and $u_{f}$ are the displacements of the structure and acoustic fluid at the couple interface.

According to the displacement and pressure continuity condition, $\mathbf{F}_{f}$ and $\mathbf{F}_{s}$ can be rewritten as: 


$$
\begin{gathered}
\mathbf{F}_{f}=\rho \int_{\partial \Omega_{s f}} \mathbf{N}_{f}{ }^{\mathrm{T}} \ddot{u}_{f} d \Gamma=\rho\left(\int_{\partial \Omega_{s f}} \mathbf{N}_{f}{ }^{\mathrm{T}} n_{f} \mathbf{N}_{s} d \Gamma\right) \ddot{\mathbf{u}}_{s} \\
\mathbf{F}_{s}=-\int_{\partial \Omega_{s f}} \mathbf{N}_{s}^{\mathrm{T}} \tau \mathrm{d} \Gamma=\int_{\partial \Omega_{s f}} \mathbf{N}_{s}^{\mathrm{T}} p \mathbf{n} \mathrm{d} \Gamma=\left(\int_{\partial \Omega_{s f}} \mathbf{N}_{s}{ }^{\mathrm{T}} \mathbf{n}_{f} \mathbf{N}_{f} \mathrm{~d} \Gamma\right) \mathbf{p}
\end{gathered}
$$

By introducing a space coupling matrix $\mathbf{H}$, Eq. (13) can be simplified:

$$
\begin{gathered}
\mathbf{H}=\int_{\partial \Omega_{s f}} \mathbf{N}_{s}^{\mathrm{T}} \mathbf{n}_{f} \mathbf{N}_{f} \mathrm{~d} \Gamma \\
\mathbf{F}_{f}=\rho \mathbf{H}^{\mathrm{T}} \ddot{\mathbf{u}}_{s}=-\rho \omega^{2} \mathbf{H}^{\mathrm{T}} \mathbf{u}_{s} \\
\mathbf{F}_{S}=\mathbf{H p}
\end{gathered}
$$

By combination of Eqs. (2), (7) and (12), the dynamic equilibrium equation of structuralacoustic non-damping system can be obtained:

$$
\left[\begin{array}{cc}
\mathbf{M}_{s} & 0 \\
-\rho \mathbf{H}^{\mathrm{T}} & \mathbf{M}
\end{array}\right]\left\{\begin{array}{c}
\ddot{\mathbf{u}} \\
\ddot{\mathbf{p}}
\end{array}\right\}+\left[\begin{array}{cc}
\mathbf{K}_{s} & -\mathbf{H} \\
0 & \mathbf{K}_{f}
\end{array}\right]\left\{\begin{array}{c}
\mathbf{u}_{s} \\
\mathbf{p}
\end{array}\right\}=\left\{\begin{array}{c}
\mathbf{F}_{e} \\
\mathbf{F}_{q}
\end{array}\right\}
$$

Considering the harmony excitation, Eq. (17) can be rewritten:

$$
\left[\begin{array}{cc}
\mathbf{K}_{s}-\omega^{2} \mathbf{M}_{s} & -\mathbf{H} \\
-\rho \omega^{2} \mathbf{H}^{\mathrm{T}} & \mathbf{K}_{f}-\omega^{2} \mathbf{M}_{f}
\end{array}\right]\left\{\begin{array}{c}
\mathbf{u}_{s} \\
\mathbf{p}
\end{array}\right\}=\left\{\begin{array}{c}
\mathbf{F}_{e} \\
\mathbf{F}_{q}
\end{array}\right\}
$$

\section{Analysis of multi-stopband laminate acoustic metamaterials for elastic wave absorption}

\subsection{Dispersion analysis and multi-frequency stopband generation}

In this section, the multi-stopband laminate acoustic metamaterials are proposed based on the 2-DOF multi-frequency vibration absorbers in Fig. 2. The laminate acoustic metamaterials consist of one basic laminate and a periodic array of multi-frequency mass-spring subsystems, the working unit cell is shown in Fig. 3. Unlike conventional vibration absorber, two masses $m$ ${ }_{1}=60 \mathrm{~g}$ and $m_{2}=0.05 m_{1}[17]=3 \mathrm{~g}$ with different spring constants $k_{1}$ and $k_{2}$ are attached to the basic 
laminate, and $\frac{1}{2 \pi} \sqrt{\frac{k_{1}+k_{2}}{m_{1}}}=\frac{1}{2 \pi} \sqrt{\frac{k_{2}}{m_{2}}}=400 \mathrm{~Hz}$. The edge lengths of the laminate in the $x, y$ and $z$ directions are $2 a=0.15 \mathrm{~m}, 2 b=0.15 \mathrm{~m}$, and $h=6.4 \mathrm{~mm}$ respectively. The displacements of the primary and secondary masses are denoted by $u_{1}^{\prime}$ and $u_{2}^{\prime}$. T300/5208 is selected as the material of the laminate: $E_{1}=181 \mathrm{GPa}, E_{2}=10.3 \mathrm{GPa}, E_{3}=10.3 \mathrm{GPa}, \mu_{12}=0.28, \rho=1600 \mathrm{~kg} / \mathrm{m}^{3}, G_{12}=7.17 \mathrm{GPa}$, $G_{13}=7.17 \mathrm{GPa}, G_{23}=3.87 \mathrm{Gpa}, X_{\mathrm{t}}=1500 \mathrm{MPa}, X_{\mathrm{c}}=1500 \mathrm{MPa}, Y_{\mathrm{t}}=40 \mathrm{MPa}, Y_{\mathrm{c}}=246 \mathrm{MPa}, S=68 \mathrm{MPa}$.

As shown in Fig. 4, the mid-plane forces can be derived as follows:

$$
\left\{\mathbf{N}_{x}\right\}=[\mathbf{A}]\left\{\boldsymbol{\varepsilon}_{x}^{0}\right\}+[\mathbf{B}]\left\{\mathbf{k}_{x}\right\}
$$

where:

$$
\begin{aligned}
& {[\mathbf{A}]=\sum_{k=1}^{n}\left([\overline{\mathbf{K}}]_{k}\left(z_{k}-z_{k-1}\right)\right)} \\
& {[\mathbf{B}]=\frac{1}{2} \sum_{k=1}^{n}\left([\overline{\mathbf{K}}]_{k}\left(z_{k}^{2}-z_{k-1}^{2}\right)\right)}
\end{aligned}
$$

Similarly, the mid-plane moments can be expressed in this way:

$$
\left\{\mathbf{M}_{x}\right\}=[\mathbf{B}]\left\{\boldsymbol{\varepsilon}_{x}^{0}\right\}+[\mathbf{D}]\left\{\mathbf{k}_{x}\right\}
$$

where:

$$
\begin{aligned}
& {[\mathbf{B}]=\frac{1}{2} \sum_{k=1}^{n}\left([\overline{\mathbf{K}}]_{k}\left(z_{k}^{2}-z_{k-1}^{2}\right)\right)} \\
& {[\mathbf{D}]=\frac{1}{3} \sum_{k=1}^{n}\left([\overline{\mathbf{K}}]_{k}\left(z_{k}^{3}-z_{k-1}^{3}\right)\right)}
\end{aligned}
$$

Subsequently, we can assume that $u_{x x}=\frac{\partial^{2} u}{\partial x^{2}} u_{x y}=\frac{\partial^{2} u}{\partial x \partial y}$. According to Eq. (21), we can redefine the moments by using $M_{1}, M_{2}, M_{6}, Q_{1}$ and $Q_{2}$ instead of $M_{x}, M_{y}, M_{x y}, Q_{x}$ and $Q_{y}$ : 


$$
\begin{aligned}
& M_{1}=\left[B_{11} u_{x}^{0}+B_{12} v_{y}^{0}+B_{16}\left(u_{y}^{0}+v_{x}^{0}\right)\right]-\left(D_{11} w_{x x}+D_{12} w_{y y}+2 D_{16} w_{x y}\right) \\
& M_{2}=\left[B_{12} u_{x}^{0}+B_{22} v_{y}^{0}+B_{26}\left(u_{y}^{0}+v_{x}^{0}\right)\right]-\left(D_{12} w_{x x}+D_{22} w_{y y}+2 D_{26} w_{x y}\right) \\
& M_{6}=\left[B_{16} u_{x}^{0}+B_{26} v_{y}^{0}+B_{66}\left(u_{y}^{0}+v_{x}^{0}\right)\right]-\left(D_{16} w_{x x}+D_{26} w_{y y}+2 D_{66} w_{x y}\right) \\
& Q_{1}=M_{1 x}+M_{6 y} \\
& Q_{2}=M_{6 x}+M_{2 y}
\end{aligned}
$$

where $Q_{1}$ and $Q_{2}$ represent the vertical force resultants, and $w$ is the displacement of the laminate in the $z$-axis.

Considering the excellent properties of symmetric laminates accompanied with the avoidance of coupling effects, the laminate is set as $[0 / 45 / 90 /-45]_{s}$. Due to the symmetry, $B_{i j}=0$, Eq. (23) can be simplified as follows:

$$
\begin{aligned}
& M_{1}=-\left(D_{11} w_{x x}+D_{12} w_{y y}+2 D_{16} w_{x y}\right) \\
& M_{2}=-\left(D_{12} w_{x x}+D_{22} w_{y y}+2 D_{26} w_{x y}\right) \\
& M_{6}=-\left(D_{16} w_{x x}+D_{26} w_{y y}+2 D_{66} w_{x y}\right) \\
& Q_{1}=M_{1 x}+M_{6 y} \\
& Q_{2}=M_{6 x}+M_{2 y}
\end{aligned}
$$

It is known that the variation of kinetic energy $\delta T$, elastic strain energy $\delta U$ and nonconservative work $\delta W_{n c}$ of the laminate can be expressed as:

$$
\delta T=\int_{-a}^{a} \int_{-b}^{b}(\rho t \ddot{w} \delta w) d x d y
$$




$$
\begin{aligned}
& \delta U=\int_{-a}^{a} \int_{-b}^{b} \int_{-t / 2}^{t / 2}\left(\sigma_{x} \delta \varepsilon_{x}+\sigma_{y} \delta \varepsilon_{y}+\sigma_{x y} \delta \varepsilon_{x y}\right) d z d x d y \\
& =\int_{-a}^{a} \int_{-b}^{b} \int_{-t / 2}^{t / 2}\left(-M_{1 x x} \delta w-M_{2 y y} \delta w-M_{6 x y} \delta w\right) d x d y \\
& +\int_{-b}^{b}\left\{\left.\left[-M_{1} \delta w_{x}+\left(M_{1 x}+M_{6 y}\right) \delta w-M_{6} \delta w_{y}\right]\right|_{x=-a} ^{x=0^{-}}+\left.\left[-M_{1} \delta w_{x}+\left(M_{1 x}+M_{6 y}\right) \delta w-M_{6} \delta w_{y}\right]\right|_{x=0^{+}} ^{x=a}\right\} d y \\
& +\int_{-a}^{a}\left\{\left.\left[-M_{2} \delta w_{y}+\left(M_{2 y}+M_{6 x}\right) \delta w-M_{6} \delta w_{x}\right]\right|_{y=-b} ^{y=0^{-}}+\left.\left[-M_{2} \delta w_{y}+\left(M_{2 y}+M_{6 x}\right) \delta w-M_{6} \delta w_{x}\right]\right|_{x=0^{+}} ^{x=b}\right\} d x \\
& \delta W_{n c}=\left.\int_{-b}^{b}\left[-M_{1} \delta w_{x}+Q_{1} \delta w-M_{6} \delta w_{y}\right]\right|_{x=-a} ^{x=a} d y \\
& +\left.\int_{-a}^{a}\left[-M_{2} \delta w_{y}+Q_{2} \delta w-M_{6} \delta w_{x}\right]\right|_{y=-b} ^{y=b} d x+k\left(u_{1}^{\prime}-w_{0}\right) \delta w_{0}
\end{aligned}
$$

The governing equation for a unit cell of infinite laminate acoustic metamaterials can be obtained according to Hamilton's principle:

$$
\int_{0}^{t}\left(\delta T-\delta U+\delta W_{n c}\right) d t=0
$$

Substituting Eqs. (25-27) into Eq. (28)

$$
\begin{aligned}
& \int_{0}^{t}\left\{\int_{-a}^{a} \int_{-b}^{b}\left(-\rho h \ddot{w}+M_{1 x x}+M_{2 y y}+2 M_{6 x y}+\left[\bar{Q}+k\left(u_{1}^{\prime}-w_{0}\right)\right] \delta(x, y)\right) \delta w d x d y\right\} d t=0 \\
& \bar{Q}=\left(Q_{1}^{x=\varepsilon_{x} / 2}-Q_{1}^{x=-\varepsilon_{x} / 2}\right) \varepsilon_{y}+\left(Q_{2}^{y=\varepsilon_{y} / 2}-Q_{1}^{y=-\varepsilon_{y} / 2}\right) \varepsilon_{x}, \varepsilon_{x}, \varepsilon_{y} \approx 0
\end{aligned}
$$

where $\delta(x, y)$ is a 2D Dirac delta function, and $\bar{Q}$ represents the discontinuity of the internal transverse shear force at the absorber location.

By setting the coefficient of $\delta w$ to zero, the governing equation of the laminate is obtained:

$$
-\rho h \ddot{w}+M_{1 x x}+M_{2 y y}+2 M_{6 x y}+\left[\bar{Q}+k\left(u_{1}^{\prime}-w_{0}\right)\right] \delta(x, y)=0
$$

It is assumed that the laminate as a rigid body moves with the average acceleration on the area $2 a \times 2 b$. Furthermore, the laminate is subjected to the concentrated force from the absorber and the lateral shear force on the four edges. Therefore, $\bar{Q}$ can be omitted. Then Eq. (30) can be integrated over the laminate: 


$$
\begin{aligned}
& \int_{-a}^{a} \int_{-b}^{b}\left\{-\rho h \ddot{w}+M_{1 x x}+M_{2 y y}+2 M_{6 x y}+\left[\bar{Q}+k\left(u_{1}^{\prime}-w_{0}\right)\right] \delta(x, y)\right\} d x d y \\
& =\int_{-a}^{a} \int_{-b}^{b}(-\rho h \ddot{w}) d y d x+\left.\int_{-a}^{a}\left(M_{2 y}+M_{6 x}\right)\right|_{y=-b} ^{y=b} d x \\
& \quad+\left.\int_{-b}^{b}\left(M_{1 x}+M_{6 y}\right)\right|_{x=-a} ^{x=a} d y+k\left(u_{1}^{\prime}-w_{0}\right)
\end{aligned}
$$

Based on Newton's Second Law, the governing equations of the primary and secondary vibration absorbers are derived as follows:

$$
\begin{gathered}
m_{1} \ddot{u}_{1}^{\prime}=k_{1}\left(w_{0}-u_{1}^{\prime}\right)+k_{2}\left(u_{2}^{\prime}-u_{1}^{\prime}\right) \\
m_{2} \ddot{u}_{2}^{\prime}=k_{2}\left(u_{1}^{\prime}-u_{2}^{\prime}\right)
\end{gathered}
$$

If a single frequency wave propagates within infinite laminate acoustic metamaterials made of a periodic unit cell (see Fig. 4(b)), the laminate's displacement $w$ and the primary and secondary absorber's displacement $u_{1}^{\prime}$ and $u_{2}^{\prime}$ can be expressed in the following form:

$$
w=p e^{j(\alpha x+\beta y-\omega t)}, u_{1}^{\prime}=q e^{-j \omega t}, u_{2}^{\prime}=r e^{-j \omega t}
$$

Substituting Eq. (34) into Eqs. (31-33) yields a standard eigenvalue problem:

$$
\left[\begin{array}{ccc}
\frac{4 \sin (\alpha \alpha) \sin (b \beta)}{\alpha \beta}\left\{\rho h w^{2}-\left[D_{11} \alpha^{4}+2\left(D_{12}+2 D_{66}\right) \alpha^{2} \beta^{2}+D_{22} \beta^{4}+4 D_{26} \alpha \beta^{3}+4 D_{16} \alpha^{3} \beta\right]\right\}-k_{1} & k_{1} & 0 \\
k_{1} & m_{1} \omega^{2}-k_{1}-k_{2} & k_{2} \\
0 & k_{2} & m_{2} \omega^{2}-k_{2}
\end{array}\right]\left[\begin{array}{c}
p \\
q \\
r
\end{array}\right]=0
$$

To have non-zero solutions in the eigenvalue problem shown in Eq. (35), the determinant of the matrix needs to be zero. There are three real and positive solutions of $\omega$ related to $\alpha$ and $\beta$.

There are two stopbands among the three dispersion surfaces shown in Fig. 5. And the stopbands are $352-378 \mathrm{~Hz}, 442-465.5 \mathrm{~Hz}$ respectively if the resonant frequency of the primary and secondary vibration absorbers is adjusted to $400 \mathrm{~Hz}$. The dispersion analysis above is focused on 
infinite laminate acoustic metamaterials. In the following section, we use finite element model to investigate the behavior of multi-stopband of laminate acoustic metamaterials.

\subsection{Verification for the stopband generated by laminate acoustic metamaterials using FRA}

In this section, a series of numerical simulations are performed to check the validity of the multi-frequency stopband. In the numerical examples, T300/520 is chosen to be the material of the laminate: $E_{1}=181 \mathrm{GPa}, E_{2}=10.3 \mathrm{GPa}, E_{3}=10.3 \mathrm{GPa}, \mu_{12}=0.28, \rho=1600 \mathrm{~kg} / \mathrm{m}^{3}, G_{12}=7.17 \mathrm{GPa}$, $G_{13}=7.17 \mathrm{GPa}, G_{23}=3.87 \mathrm{Gpa}, X_{\mathrm{t}}=1500 \mathrm{MPa}, X_{\mathrm{c}}=1500 \mathrm{MPa}, Y_{\mathrm{t}}=40 \mathrm{MPa}, Y_{\mathrm{c}}=246 \mathrm{MPa}, S=68 \mathrm{MPa}$. To better understand how the boundary conditions and damping ratios of the vibration absorbers affect the stopband, we redo the simulation with the finite-element commercial software Hyperworks. In the simulations, the stack sequence of the symmetrical laminate (see Fig. 6(a)) is $[0 / 45 / 90 /-45]_{s}$, and the vertical length and horizontal length are $L_{a}=6 \mathrm{~m}$ and $L_{b}=3.6 \mathrm{~m}$ respectively. The thickness of each ply is set to be $h=0.8 \mathrm{~mm}$. The resonant frequencies of the primary and secondary absorber are set to be $f_{1}=f_{2}=400 \mathrm{~Hz}$. The masses of the primary and secondary absorber are set to be $m_{1}=60 \mathrm{~g}, m_{2}=0.05 m_{1}=3 \mathrm{~g}$. The whole weight of the subsystems is $20.3 \%$ of the total mass of the multi-stopband laminate acoustic metamaterials.

The unit cell (see Fig. 3) considered in the dispersion analysis is used to investigate the periodic structure. The laminate is modeled by $40 \times 24$ four-node rectangular plate elements. The 2-DOF mass-spring-damper subsystem is arranged uniformly on the node of the laminate except the first and last columns as well as the first and last rows (i.e., the blue dot in Fig. 6(b)). An external excitation with amplitude of $10 \mathrm{kN}$ in the $z$ direction is applied at the second node on the center line (i.e., the green dot in Fig. 6(b)), and the laminate is simply fixed at the two vertical edges. 
In Figures 7(a) and 7(b), we plot the frequency response functions (FRFs) of the laminate acoustic metamaterials at two representative points corresponding to different damping ratio. The black curves represent the laminate acoustic metamaterials without vibration absorbers for reference. The red curves show two stopbands between $352-378 \mathrm{~Hz}$ and $442-465.5 \mathrm{~Hz}$ with a low damping ratio $\xi_{1}=\xi_{2}=0.001$ for each vibration absorber. The blue curves show FRFs of laminate acoustic laminates with the damping ratio increasing from 0.001 to 0.1 . The purple curves show a wider continuous stopband since the low damping in the primary absorber makes the excitation energy rapidly transferred into the mass-spring-damper subsystems and the high damping in the secondary absorber suppress the vibration effectively further. The behavior of the multi-stopband agrees well with our dispersion analysis.

It is common sense that the system maximizes the coupling of external excitation under resonance. The system under resonance is prone to lose energy through resonance scattering of the vibration absorbers. By introducing the multi-frequency vibration absorbers into the laminate acoustic metamaterials, we design the multi-stopband laminate acoustic metamaterials that are proved to be capable of effectively suppressing the low-frequency vibration within the bandgaps. In addition, the bandgap can be widened with an appropriate damping ratio of the vibration absorbers, as shown in Fig. 7 (the purple curves).

\section{Numerical examples and discussion}

\subsection{The coupling model of laminate acoustic metamaterials and three-dimensional acoustic cavity}

In this section, we present some numerical results for the analysis of laminate acoustic metamaterials in structural-acoustic coupled system. Firstly, a rectangular laminate with length $0.6 \mathrm{~m}$, width $0.36 \mathrm{~m}$ and thickness $0.016 \mathrm{~m}$, which is coupled with a three-dimensional acoustic 
cavity with length $0.6 \mathrm{~m}$, width $0.36 \mathrm{~m}$ and depth $0.18 \mathrm{~m}$, is considered (see Fig. 8). The hexahedron is completely filled with air, and the acoustic parameters are defined as follows: $\rho_{f}=1.225 \mathrm{~kg} / \mathrm{m}^{3}, c=340 \mathrm{~m} / \mathrm{s}$. The mechanical parameters of the laminate are: $E_{1}=181 \mathrm{GPa}$, $E_{2}=10.3 \mathrm{GPa}, E_{3}=10.3 \mathrm{GPa}, \mu_{12}=0.28, \rho=1600 \mathrm{~kg} / \mathrm{m}^{3}, G_{12}=7.17 \mathrm{GPa}, G_{13}=7.17 \mathrm{GPa}, G_{23}=3.87 \mathrm{Gpa}$, $X_{\mathrm{t}}=1500 \mathrm{MPa}, X_{\mathrm{c}}=1500 \mathrm{MPa}, Y_{\mathrm{t}}=40 \mathrm{MPa}, Y_{\mathrm{c}}=246 \mathrm{MPa}, S=68 \mathrm{MPa}$. The point A with the coordinate of $(420,180,180)$ (i.e., the triangle dot in Fig. 8) as well as the point B with the coordinate of $(270,180,0)$ (i.e., the circle dot in Fig. 8) is selected as the structural response point and acoustic response point respectively. The two vertical edges of laminate at $x=0$ and $0.6 \mathrm{~m}$ are hinged, and the external excitation with amplitude of $1 \mathrm{kN}$ in the $z$ direction is applied at the node with the coordinate of $(30,180,180)$. The laminate is modeled by $40 \times 24$ four-node rectangular plate elements. The stack sequence of the laminate is $[0 / 45 / 90 /-45]_{s}$ and the thickness of each ply is set to be $h=2 \mathrm{~mm}$.

It is well known that only bending modes that provide a net change of the fluid volume create the interaction and the energy transfer. Thus, FRA is conducted first to find the bending mode frequency of multi-stopband laminate acoustic metamaterials without vibration absorbers. The first-order bending mode frequency is around $225 \mathrm{~Hz}$ and second-order bending mode frequency is $335 \mathrm{~Hz}$. In order to obtain the best effect of vibration isolation, we have made a parametric analysis with consideration of the ratio between the resonant frequency of multifrequency vibration absorbers and the bending mode frequency of the laminate. The design parameter for the parametric analysis is the resonant frequency $f$ of multi-frequency vibration absorbers, varying between $200 \mathrm{~Hz}$ and $250 \mathrm{~Hz}$. The chosen step $\Delta f$ is $5 \mathrm{~Hz}$. The ratio $Z$ can be denoted as: 


$$
Z=\frac{f}{F_{1}}
$$

where $F_{1}$ represents first-order bending mode frequency of the laminate.

Figure 9 shows a comparison of the vibration response of the laminate at point $A$ as the ratio changes. The vibration response curves have clearly illustrated that the big vibration around $225 \mathrm{~Hz}$ has been suppressed effectively when the ratio $Z=1.022$ (i.e., red curve in Fig. 9). Hence the resonant frequencies of the primary and secondary absorber are set to be $f_{1}=f_{2}=230 \mathrm{~Hz}$. The masses and damping ratios of the primary and secondary absorber are set to be $m_{1}=6 \mathrm{~g}$, $\xi_{1}=0.1$ and $m_{2}=0.05 m_{1}=0.3 \mathrm{~g}, \xi_{2}=0.5$ respectively.

In Figures 10(a) and 10(b), we plot the frequency response functions (FRFs) of the laminate acoustic metamaterials at two representative points in structural-acoustic coupled system. Figure 10(a) presents the vibration response in laminate with and without vibration absorber. And Figure 10(b) indicates the sound pressure level in the acoustic cavity with and without vibration absorber. The black curves represent the laminate acoustic metamaterials without vibration absorbers for reference. The red curves show that the vibration and noise around the $225 \mathrm{~Hz}$ have been effectively reduced. In other words, this is due to the existence of the two stopbands around the $225 \mathrm{~Hz}$.

\subsection{The coupling analysis of front panel and passenger compartment cavity of vehicle}

The vibration and sound pressure level response of the coupling system of front panel and passenger compartment cavity is investigated in this section. The design of the vehicle's front panel has a significant impact on $\mathrm{NVH}$ performance of vehicle. It is due to the fact that the front panel is close to the engine; the engine transmits the vibration to the front panel, thereby radiating noise inside the vehicle. In addition, the front panel can block the noise between the 
engine and passenger compartment cavity. Therefore, the proper design of the front panel is very important in the vehicle development process.

In this example, frequency response analysis (FRA) is performed by using the structuralacoustic interaction model (as shown in Fig. 11) to investigate the performance of laminate acoustic metamaterials. The geometric parameters of the front panel are: length $L_{\mathrm{a}}=1150 \mathrm{~mm}$, width $L_{\mathrm{b}}=390 \mathrm{~mm}$ and thickness $h=8 \mathrm{~mm}$. The resonant frequencies of the primary and secondary absorber are both set to be $f_{1}=f_{2}=300 \mathrm{~Hz}$. The masses and damping ratios of the primary and secondary absorber are set to be $m_{1}=20 \mathrm{~g}, \xi_{1}=0.1$ and $m_{2}=0.05 m_{1}=1 \mathrm{~g}, \xi_{2}=0.5$ respectively. The material parameters of the front panel here are the same as those used in the first example. The acoustic parameters of the passenger compartment cavity are expressed as follows: $\rho_{f}=1.225 \mathrm{~kg} / \mathrm{m}^{3}, c=340 \mathrm{~m} / \mathrm{s}$. For this case, the front panel composed of laminate acoustic materials is segmented into 430 rectangular elements, and the fluid domain is divided into 25351 tetrahedral elements. The front panel is surrounded by a fixed support. The external excitation with amplitude of $10 \mathrm{kN}$ in the $z$ direction is applied at the center of front panel. The response point is selected at the center of the front panel and the right ear of driver in the passenger compartment cavity (i.e., the magenta dot in Fig. 11).

The results for front panel comprising vibration absorbers are depicted in Figures 12(a) and 12(b) as red dotted curves. The black curves are plotted for the front panel without vibration absorbers. In Figure 12(b), it is seen that the sound pressure level response is reduced obviously around $300 \mathrm{~Hz}$, which agrees remarkably well with the vibration response results shown in Figure 12(a). As expected, the front panel composed of multi-stopband laminate acoustic metamaterials shows the excellent $\mathrm{NVH}$ performance in the actual application. 


\section{Conclusions}

In this paper, a new multi-stopband laminate acoustic metamaterial designed by attaching a periodic array of mass-spring-damper subsystems has been presented. Based on the concept of two degrees of freedom (2-DOF) multi-frequency vibration absorber, the band gap of multistopband laminate acoustic metamaterials composed of carbon-fiber-reinforced polymer (CFRP) has been derived by dispersion analysis in this work. Numerical results reveal that two bandgaps are generated around the absorbers' resonant frequency. To check the validity of the multifrequency stopband, finite-element modeling and frequency response analysis are performed. According to the results, it is seen that the excitation energy is rapidly transferred to the absorbers due to the low damping in the primary absorber, on the other hand, the high damping in the secondary absorber expands the original two stopbands to a wider stopband and inhibits the excitation energy effectively. In the analysis of laminate acoustic metamaterials for structural-acoustic coupled system, the excellent Noise Vibration and Harshness (NVH) performance of laminate acoustic metamaterials has been clearly verified.

\section{Acknowledgement}

The project is supported by the Project funded by China Postdoctoral Science Foundation. The authors also wish to thank Research Project of the Science Fund of State Key Laboratory of Advanced Design and Manufacturing for Vehicle Body (Grant No. 51375001 and 31615002), Research Project of State Key Laboratory of Mechanical Systems and Vibration (MSV 201613 and MSV201711), and Open Foundation of the State Key Laboratory of Fluid Power and Mechatronic Systems GZK F-201601. 


\section{References}

[1] Veselago VG. The electrodynamics of substances with simultaneously negative values of and $\mu$. Soviet physics uspekhi. 1968;10(4):509.

[2] Walser ARM. Electromagnetic metamaterials. Proceedings of SPIE - The International Society for Optical Engineering. 2001;33(10):931-4.

[3] Nefedov IS, Tretyakov SA. Photonic band gap structure containing metamaterial with negative permittivity and permeability. Physical Review E Statistical Nonlinear \& Soft Matter Physics. 2002;66(3 Pt 2B):036611.

[4] Sigalas MM, Economou EN. Elastic and acoustic wave band structure. Journal of sound and vibration. 1992;158(2):377-82.

[5] Kushwaha MS, Halevi P, Dobrzynski L, Djafarirouhani B. Acoustic band structure of periodic elastic composites. Physical Review Letters. 1993;10(9):977-1094.

[6] Sigalas M, Economou EN. Band structure of elastic waves in two dimensional systems. Solid State Communications. 1993;86(3):141-3.

[7] Li E, He ZC, Wang G. An exact solution to compute the band gap in phononic crystals. Computational Materials Science. 2016;122:72-85.

[8] Li E, He ZC, Hu JY, Long XY. Volumetric locking issue with uncertainty in the design of locally resonant acoustic metamaterials. Comput Method Appl M. 2017;324:128-48.

[9] Li E, He ZC, Wang G, Liu GR. An ultra-accurate numerical method in the design of liquid phononic crystals with hard inclusion. Comput Mech. 2017.

[10] Li E, He ZC, Wang G, Liu GR. An efficient algorithm to analyze wave propagation in fluid/solid and solid/fluid phononic crystals. Comput Method Appl M. 2018;333:421-42.

[11] Liu Z, Zhang X, Mao Y, Zhu YY, Yang Z, Chan CT, et al. Locally resonant sonic materials. Science. 2000;289(5485):1734-6.

[12] He ZC, Li E, Wang G, Li GY, Xia Z. Development of an efficient algorithm to analyze the elastic wave in acoustic metamaterials. Acta Mechanica. 2016;227(10):3015-30.

[13] Liu Z, Chan CT, Sheng P. Analytic model of phononic crystals with local resonances. Physical Review B. 2005;71(1):014103.

[14] Sheng P, Zhang XX, Liu Z, Chan CT. Locally resonant sonic materials. Physica B: Condensed Matter. 2003;338(1):201-5. 
[15] Yang Z, Mei J, Yang M, Chan NH, Sheng P. Membrane-type acoustic metamaterial with negative dynamic mass. Physical review letters. 2008;101(20):204301.

[16] Lee SH, Park CM, Seo YM, Wang ZG, Kim CK. Acoustic metamaterial with negative density. Physics letters A. 2009;373(48):4464-9.

[17] Peng H, Pai PF, Deng H. Acoustic multi-stopband metamaterial plates design for broadband elastic wave absorption and vibration suppression. International Journal of Mechanical Sciences. 2015;103:104-14.

[18] Fang N, Xi D, Xu J, Ambati M, Srituravanich W, Sun C, et al. Ultrasonic metamaterials with negative modulus. Nature Materials. 2006;5(6):452-6.

[19] Wu Y, Lai Y, Zhang Z-Q. Elastic metamaterials with simultaneously negative effective shear modulus and mass density. Physical review letters. 2011;107(10):105506.

[20] Lai Y, Wu Y, Sheng P, Zhang ZQ. Hybrid elastic solids. Nature Materials. 2011;10(8):620-4.

[21] Li J, Chan CT. Double-negative acoustic metamaterial. Physical Review E. 2004;70(5):055602.

[22] Ding Y, Liu Z, Qiu C, Shi J. Metamaterial with simultaneously negative bulk modulus and mass density. Physical Review Letters. 2007;99(9):093904.

[23] Lee SH, Park CM, Seo YM, Wang ZG, Kim CK. Composite acoustic medium with simultaneously negative density and modulus. Physical Review Letters. 2010;104(5):054301.

[24] Yang M, Ma G, Yang Z, Sheng P. Coupled membranes with doubly negative mass density and bulk modulus. Physical review letters. 2013;110(13):134301.

[25] Zhou X, Hu G. Superlensing effect of an anisotropic metamaterial slab with near-zero dynamic mass. Applied Physics Letters. 2011;98(26):263510.

[26] Zhang S, Yin L, Fang N. Focusing ultrasound with an acoustic metamaterial network. Physical review letters. 2009;102(19):194301.

[27] Li J, Fok L, Yin X, Bartal G, Zhang X. Experimental demonstration of an acoustic magnifying hyperlens. CALIFORNIA UNIV BERKELEY NANOSCALE SCIENCE AND ENGINEERING CENTER; 2009.

[28] Lai Y, Chen H, Zhang Z-Q, Chan CT. Complementary media invisibility cloak that cloaks objects at a distance outside the cloaking shell. Physical review letters. 2009;102(9):093901. 
[29] Zheng L-Y, Wu Y, Ni X, Chen Z-G, Lu M-H, Chen Y-F. Acoustic cloaking by a nearzero-index phononic crystal. Applied Physics Letters. 2014;104(16):161904.

[30] Wei Q, Cheng Y, Liu X-j. Acoustic total transmission and total reflection in zero-index metamaterials with defects. Applied Physics Letters. 2013;102(17):174104.

[31] Luo J, Lai Y. Anisotropic zero-index waveguide with arbitrary shapes. Scientific reports. 2014;4.

[32] Stenger N, Wilhelm M, Wegener M. Experiments on elastic cloaking in thin plates. Physical Review Letters. 2012;108(1):014301.

[33] Luo C, Johnson SG, Joannopoulos JD, Pendry JB. Subwavelength imaging in photonic crystals. Physical Review B. 2003;68(4):045115.

[34] Mei J, Ma G, Yang M, Yang Z, Wen W, Sheng P. Dark acoustic metamaterials as super absorbers for low-frequency sound. Nature communications. 2012;3:756.

[35] Wang G, Wen X, Wen J, Shao L, Liu Y. Two-dimensional locally resonant phononic crystals with binary structures. Physical Review Letters. 2004;93(15):154302.

[36] Wang G, Wen X, Wen J, Liu Y. Quasi-One-Dimensional Periodic Structure with Locally Resonant Band Gap. Journal of Applied Mechanics. 2006;73(1):págs. 167-70.

[37] Yu D, Liu Y, Wang G, Zhao H, Qiu J. Flexural vibration band gaps in Timoshenko beams with locally resonant structures. Journal of applied physics. 2006;100(12):124901.

[38] Yu D, Liu Y, Zhao H, Wang G, Qiu J. Flexural vibration band gaps in Euler-Bernoulli beams with locally resonant structures with two degrees of freedom. Physical Review B. 2006;73(6):064301.

[39] Oudich M, Assouar MB, Hou Z. Propagation of acoustic waves and waveguiding in a two-dimensional locally resonant phononic crystal plate. Applied Physics Letters. 2010;97(19):193503.

[40] Oudich M, Li Y, Assouar BM, Hou Z. A sonic band gap based on the locally resonant phononic plates with stubs. New Journal of Physics. 2010;12(8):083049.

[41] Oudich M, Senesi M, Assouar MB, Ruzenne M, Sun J-H, Vincent B, et al. Experimental evidence of locally resonant sonic band gap in two-dimensional phononic stubbed plates. Physical Review B. 2011;84(16):165136. 
[42] Claeys CC, Vergote K, Sas P, Desmet W. On the potential of tuned resonators to obtain low-frequency vibrational stop bands in periodic panels. Journal of Sound \& Vibration. 2013;332(6):1418-36.

[43] Ma Y, Scarpa F, Zhang D, Zhu B, Chen L, Hong J. A nonlinear auxetic structural vibration damper with metal rubber particles. Smart Materials \& Structures. 2013;22(8):084012.

[44] Zouari S, Genevaux JM, Brocail J, Ablitzer F. Band gap formation in thin plates with a periodic array of resonators. Mechanics \& Industry. 2017;18(3):304.

[45] He ZC, Xiao X, Li E. Design for structural vibration suppression in laminate acoustic metamaterials. Composites Part B: Engineering. 2017.

[46] Nobrega ED, Gautier F, Pelat A, Santos JMCD. Vibration band gaps for elastic metamaterial rods using wave finite element method. Mechanical Systems \& Signal Processing. 2016;79:192-202.

[47] Claeys C, Deckers E, Pluymers B, Desmet W. A lightweight vibro-acoustic metamaterial demonstrator: Numerical and experimental investigation. Mechanical Systems \& Signal Processing. 2016;s 70-71:853-80.

[48] Subhani M, Al-Ameri R. Strength reduction in square columns confined with CFRP under marine environment. Composites Part B: Engineering. 2016;97:183-92.

[49] Liu T, Hou S, Nguyen X, Han X. Energy absorption characteristics of sandwich structures with composite sheets and bio coconut core. Composites Part B: Engineering. 2017;114:328-38.

[50] Qin Y, Li YH. Influences of hygrothermal environment and installation mode on vibration characteristics of a rotating laminated composite beam. Mechanical Systems \& Signal Processing. 2017;91:23-40.

[51] Ren Y, Qiu L, Yuan S, Su Z. A diagnostic imaging approach for online characterization of multi-impact in aircraft composite structures based on a scanning spatialwavenumber filter of guided wave. Mechanical Systems \& Signal Processing. 2017;90:44-63.

[52] Kobayashi S, Tsukada T, Morimoto T. Resin Impregnation Behavior in Carbon Fiber Reinforced Polyamide 6 Composite: Effects of Yarn Thickness, Fabric Lamination and Sizing Agent. Composites Part A Applied Science \& Manufacturing. 2017;101.

[53] Galuppi L, Royer-Carfagni G. Shear coupling effects of the core in curved sandwich beams. Composites Part B: Engineering. 2015;76:320-31. 
[54] Nehete DV, Modak SV, Gupta K. Coupled vibro-acoustic model updating using frequency response functions. Mechanical Systems \& Signal Processing. 2016;s 70-71:308-19.

[55] Klaerner M, Wuehrl M, Kroll L, Marburg S. FEA-based methods for optimising structure-borne sound radiation. Mechanical Systems \& Signal Processing. 2016.

[56] Wang M, Huang Q. A new hybrid uncertain analysis method for structural-acoustic systems with random and interval parameters. Computers \& Structures. 2016;175:15-28.

[57] Jun Z, Geng-dong C, Wen-zhong Z, Guo-zhong Z. Acoustic design optimization for vibration of cylinder container coupled with interior acoustic field. Journal of Sound and Vibration. 2009;328(4):607-17.

[58] Xia B, Yu D. An interval random perturbation method for structural-acoustic system with hybrid uncertain parameters. International Journal for Numerical Methods in Engineering. 2013;97(3):181-206.

[59] Li E, He ZC, Jiang Y, Li B. 3D mass-redistributed finite element method in structuralacoustic interaction problems. Acta Mechanica. 2015:1-23.

[60] Li E, He ZC, Xu X, Liu GR. Hybrid smoothed finite element method for acoustic problems. Comput Method Appl M. 2015;283:664-88.

[61] He ZC, Li E, Li GY, Wu F, Liu GR, Nie X. Acoustic simulation using alpha-FEM with a general approach for reducing dispersion error. Eng Anal Bound Elem. 2015;61:241-53.

[62] Li E, He ZC. Stability and accuracy improvement for explicit formulation of time domain acoustic problems. Eng Anal Bound Elem. 2017;83(Supplement C):217-28. 
Figure

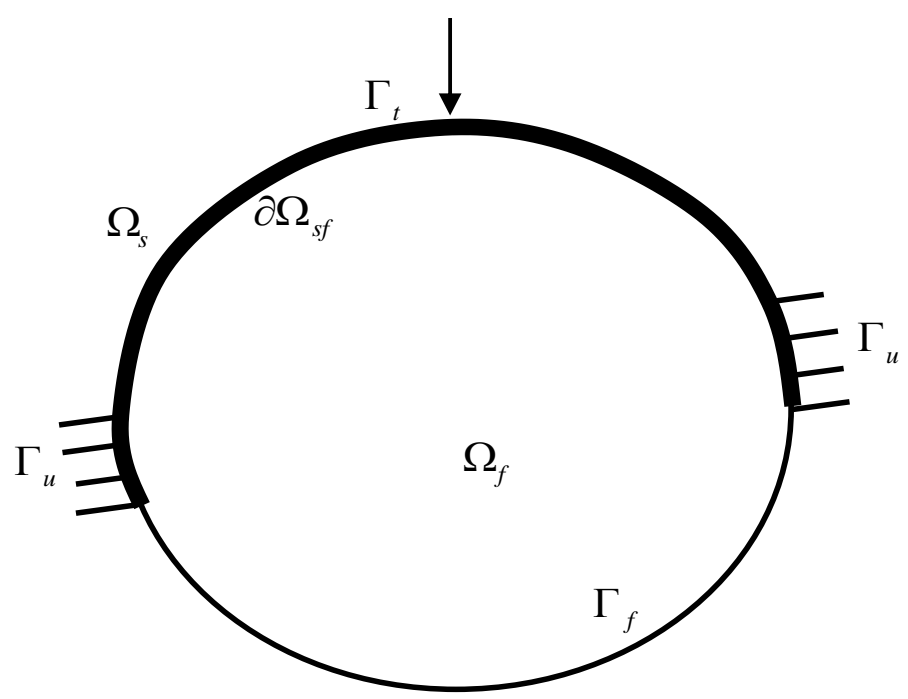

Fig. 1. A structural-acoustic system 




Fig. 2. The schematic view of the laminate metamaterial with spring-mass subsystems 


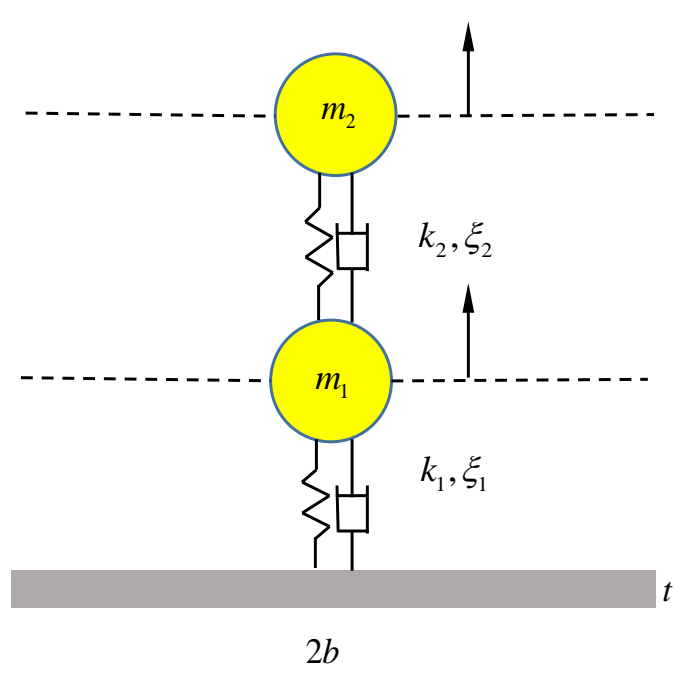

(a)

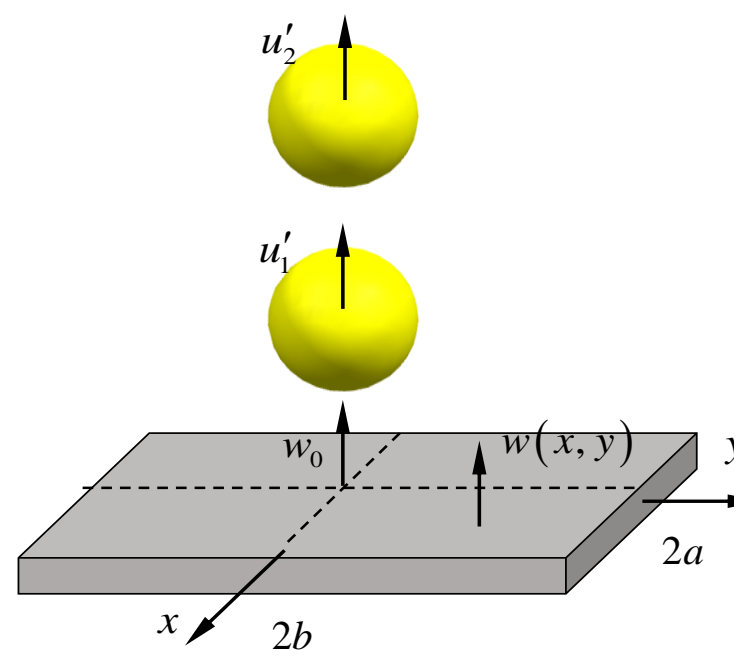

(b)

Fig. 3. A unit cell of the multi-stopband laminate metamaterial: (a) front view, and (b) perspective view 


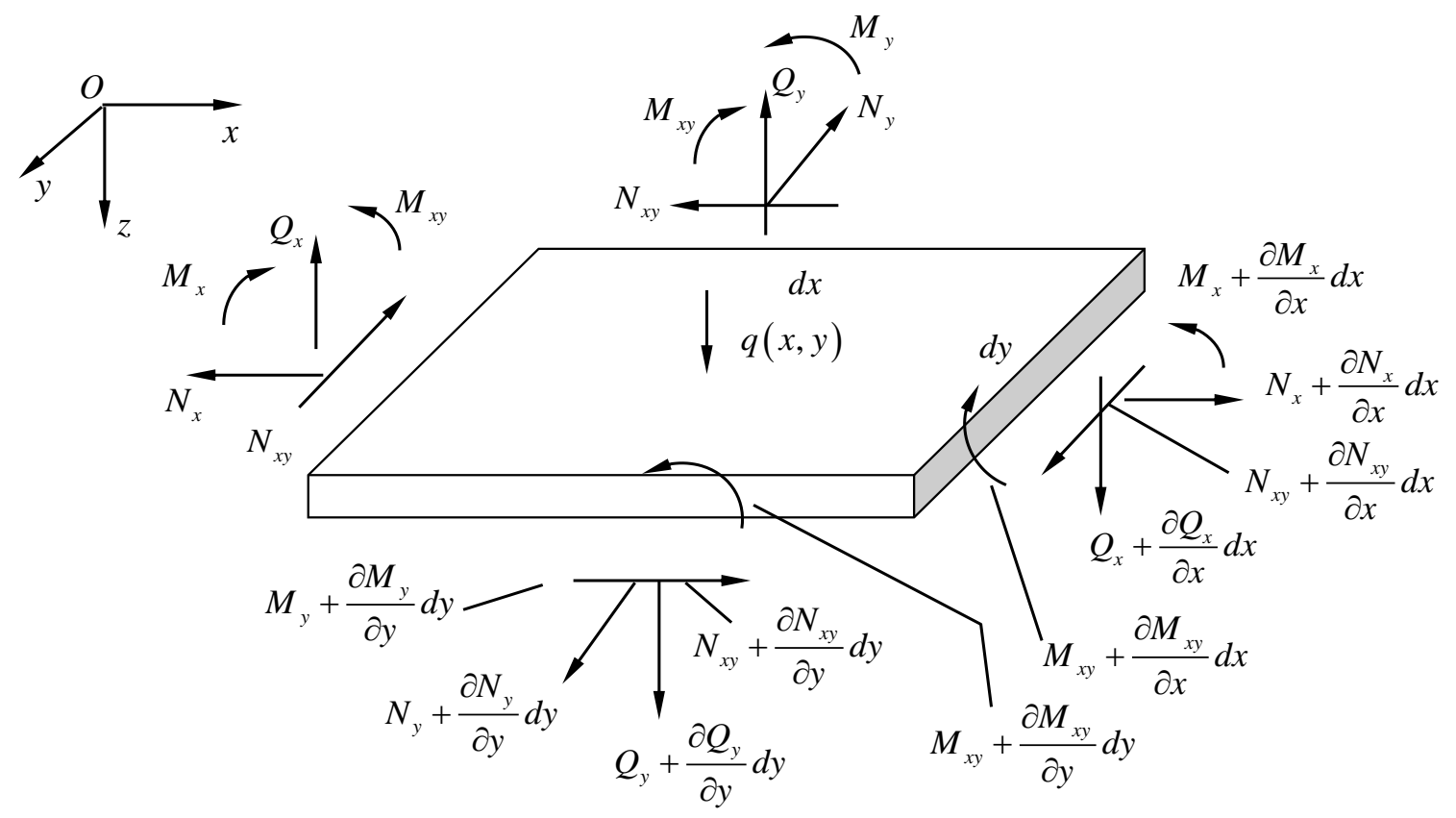

Fig. 4. The schematic view of positive Mid-Plane forces and Moments of the ply. 


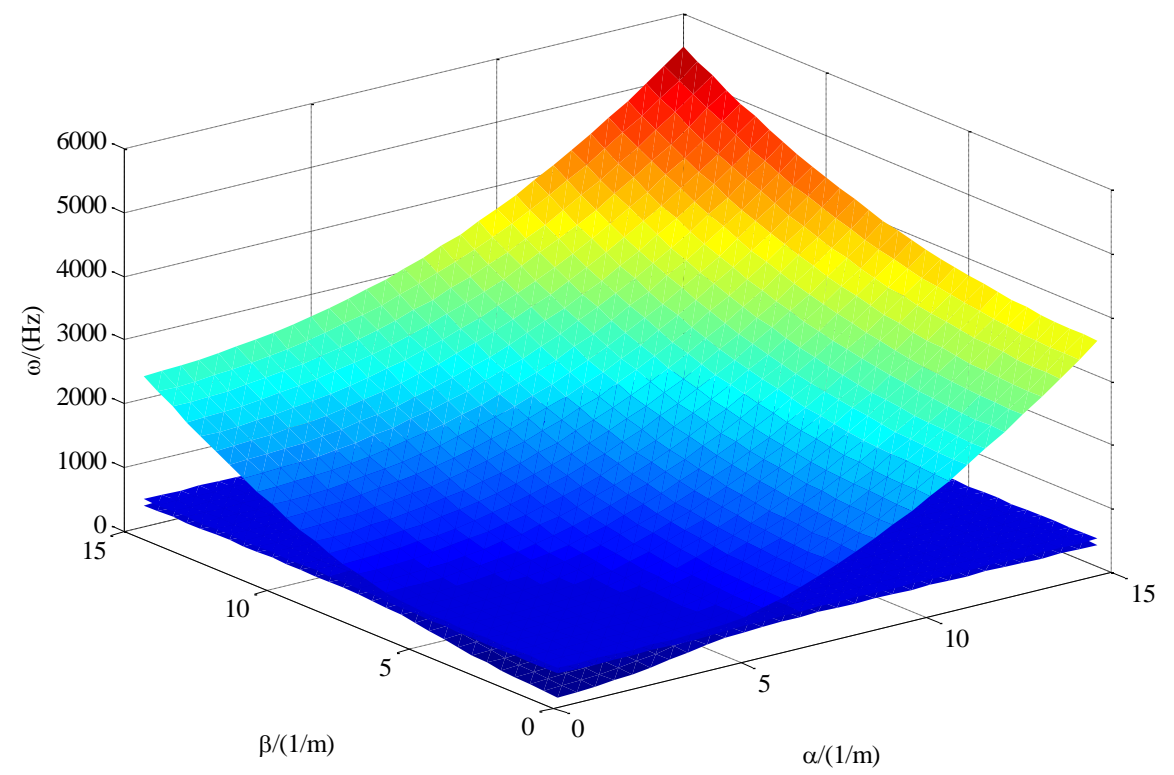

(a)

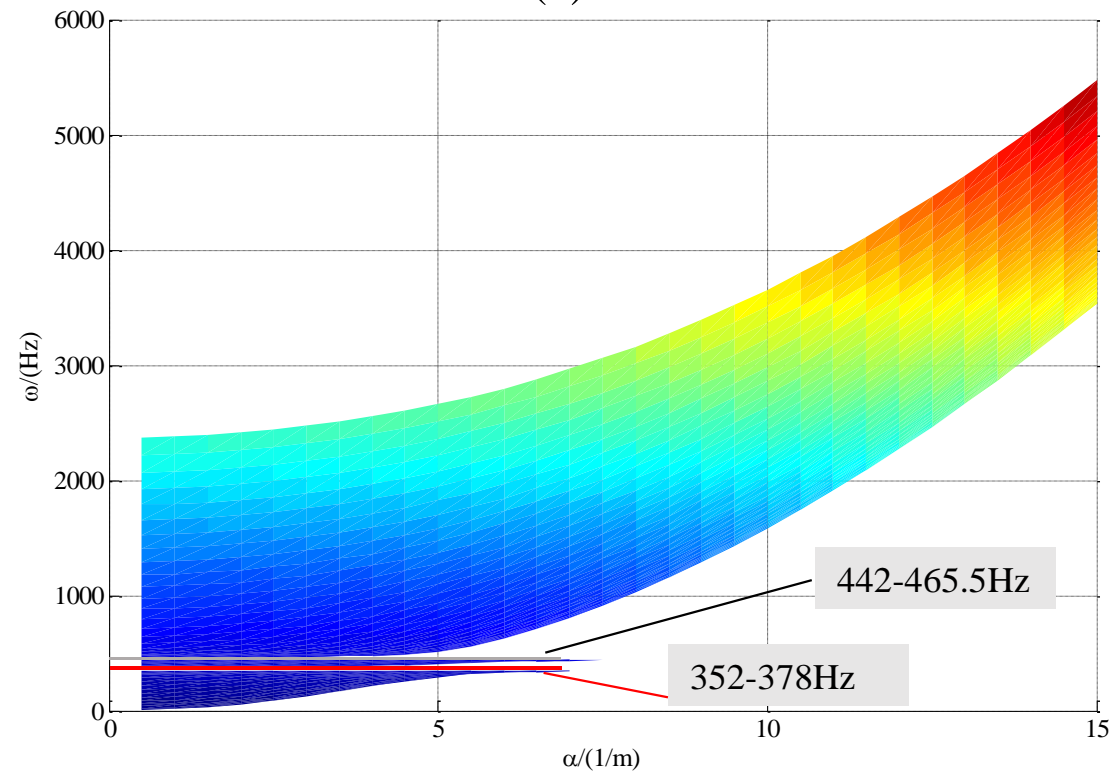

(b) 
Fig. 5. Dispersion surfaces and stopband: (a) dispersion surfaces, and (b) stopband (gray and red rectangle).

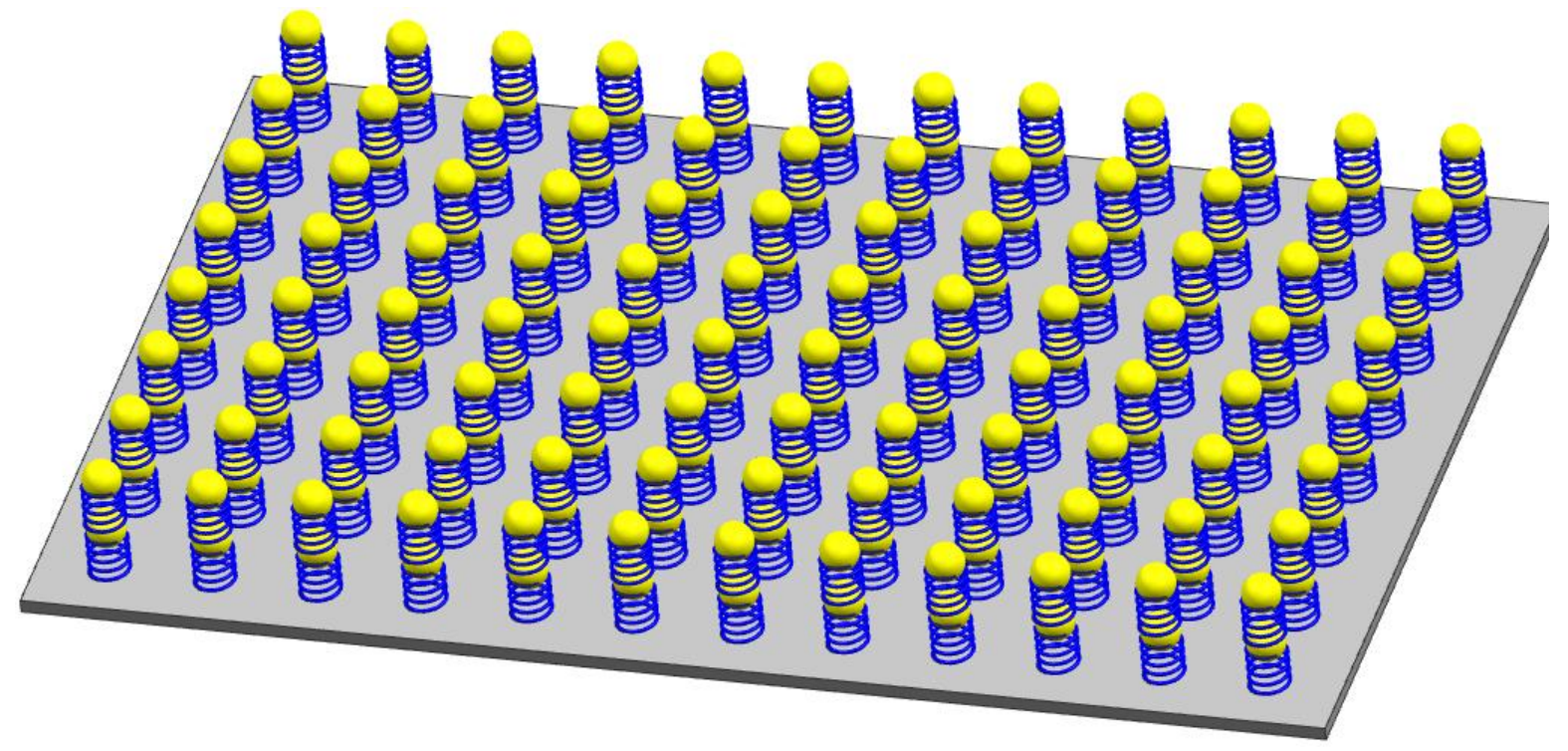

(a) 


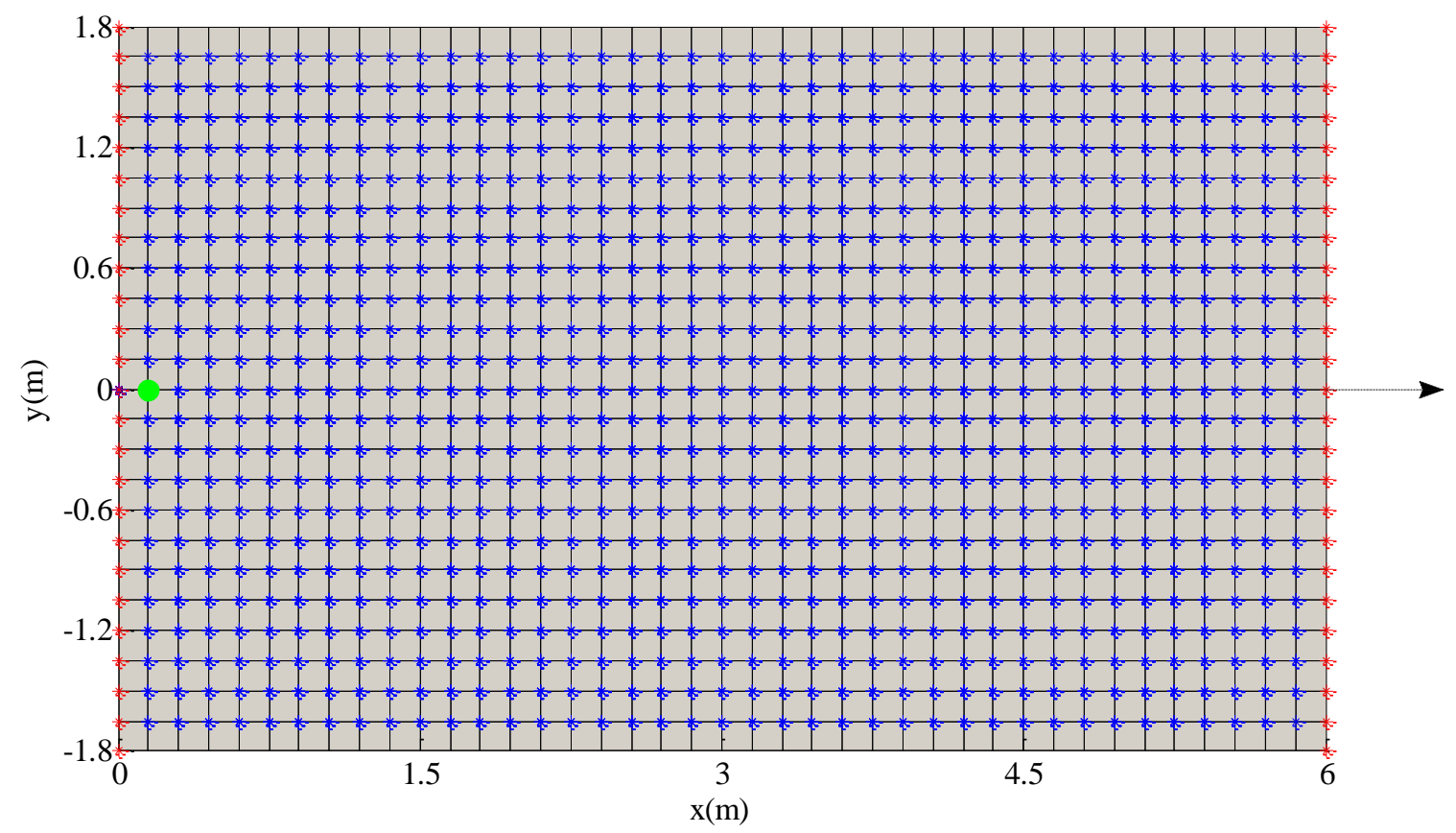

(b)

Fig. 6. A multi-stopband laminate acoustic metamaterial with two edges hinged: (a) a 3D model, and (b) a finite-element model.

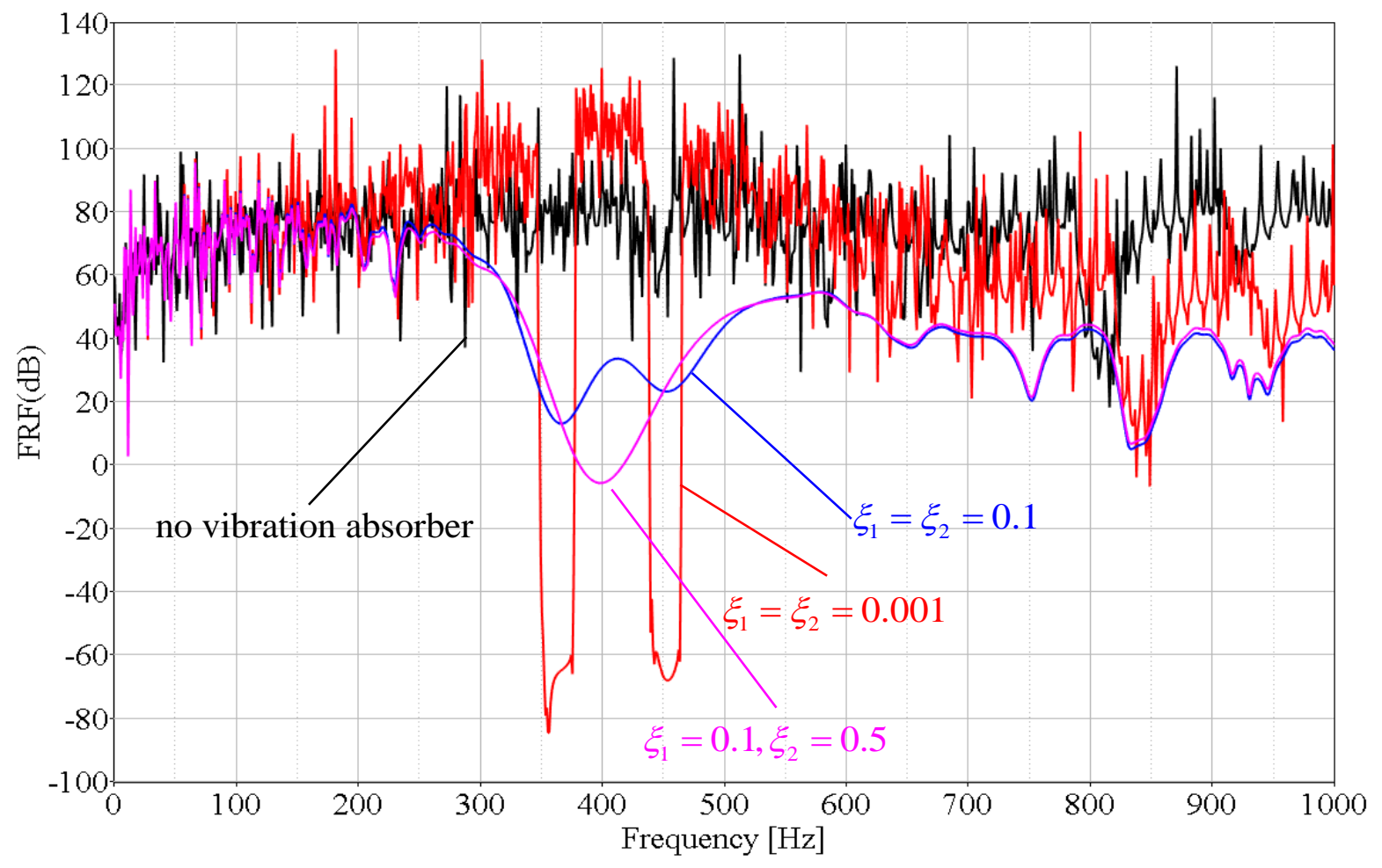


(a)

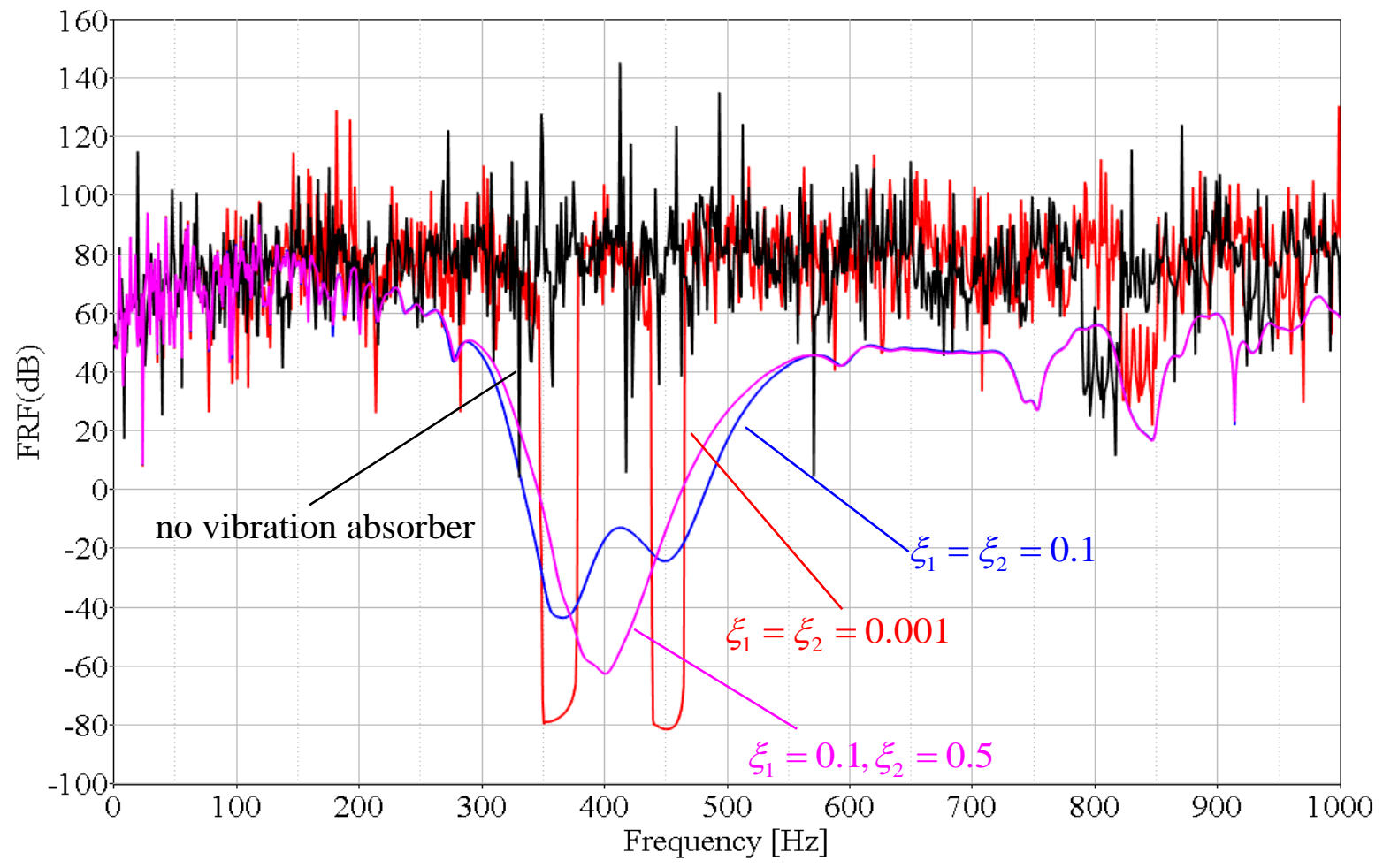

(b)

Fig. 7. Frequency response functions of the laminate acoustic metamaterial in symmetry with different damping ratios: $(a)$ response at $x=0.5 L_{\mathrm{a}}$ and $y=0.5 L_{\mathrm{b}}$, and $(b)$ response at $x=0.7 L_{\mathrm{a}}$ and $y=0.5 L_{\mathrm{b}}$

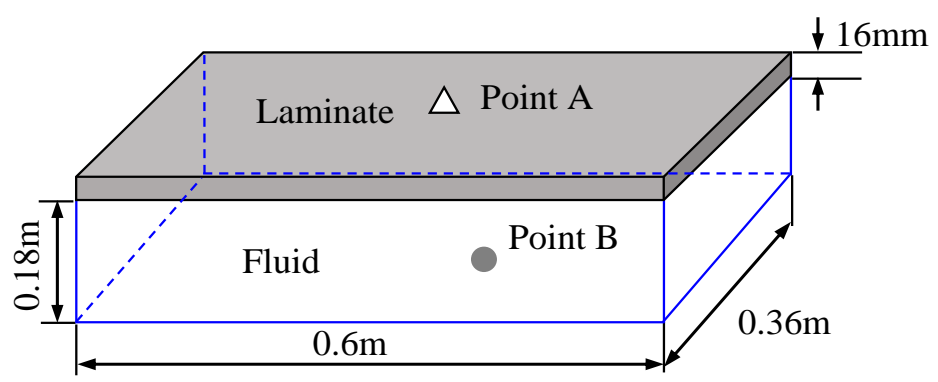

Fig. 8. Three-dimensional laminate-acoustic cavity coupling model 


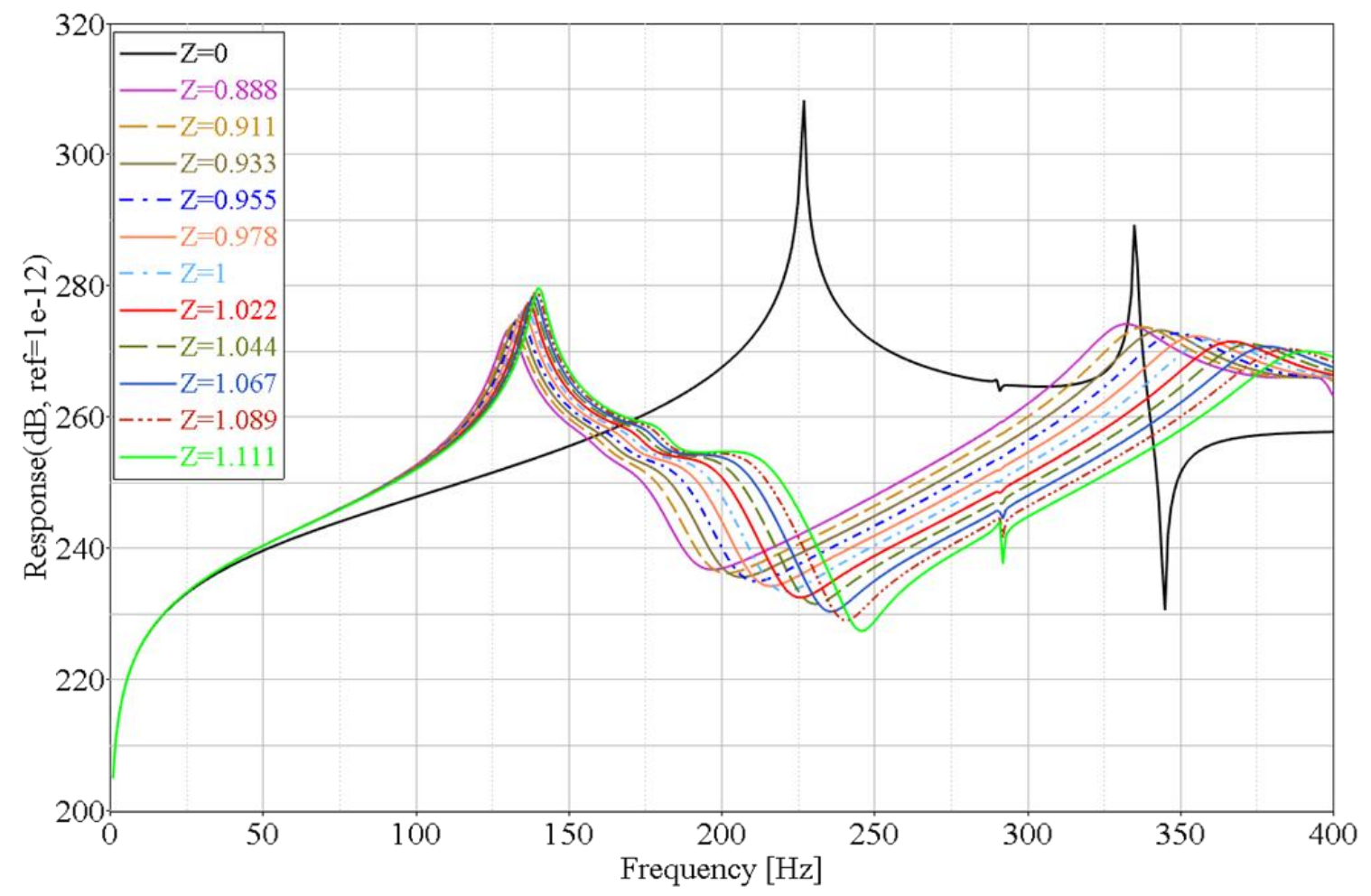

Fig. 9. Comparison of the vibration response curves with different ratios. 




(a) 


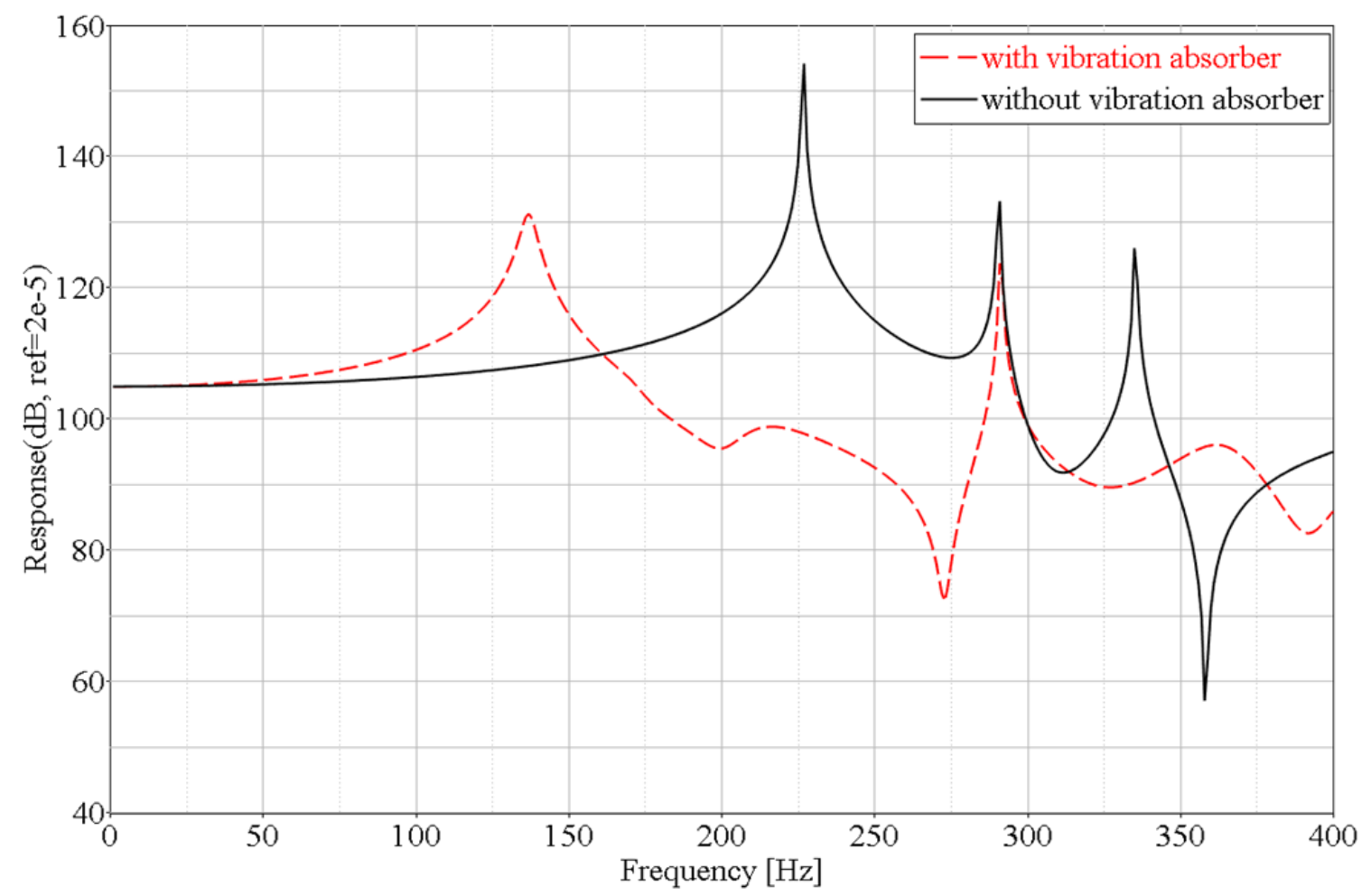

(b)

Fig. 10. Comparison of response curves in the structural-acoustic coupled system (a) vibration response at point $A$ with and without vibration absorbers, and (b) sound pressure level response at point $B$ with and without vibration absorbers



Fig. 11. The schematic view of front panel and passenger compartment cavity coupling model 


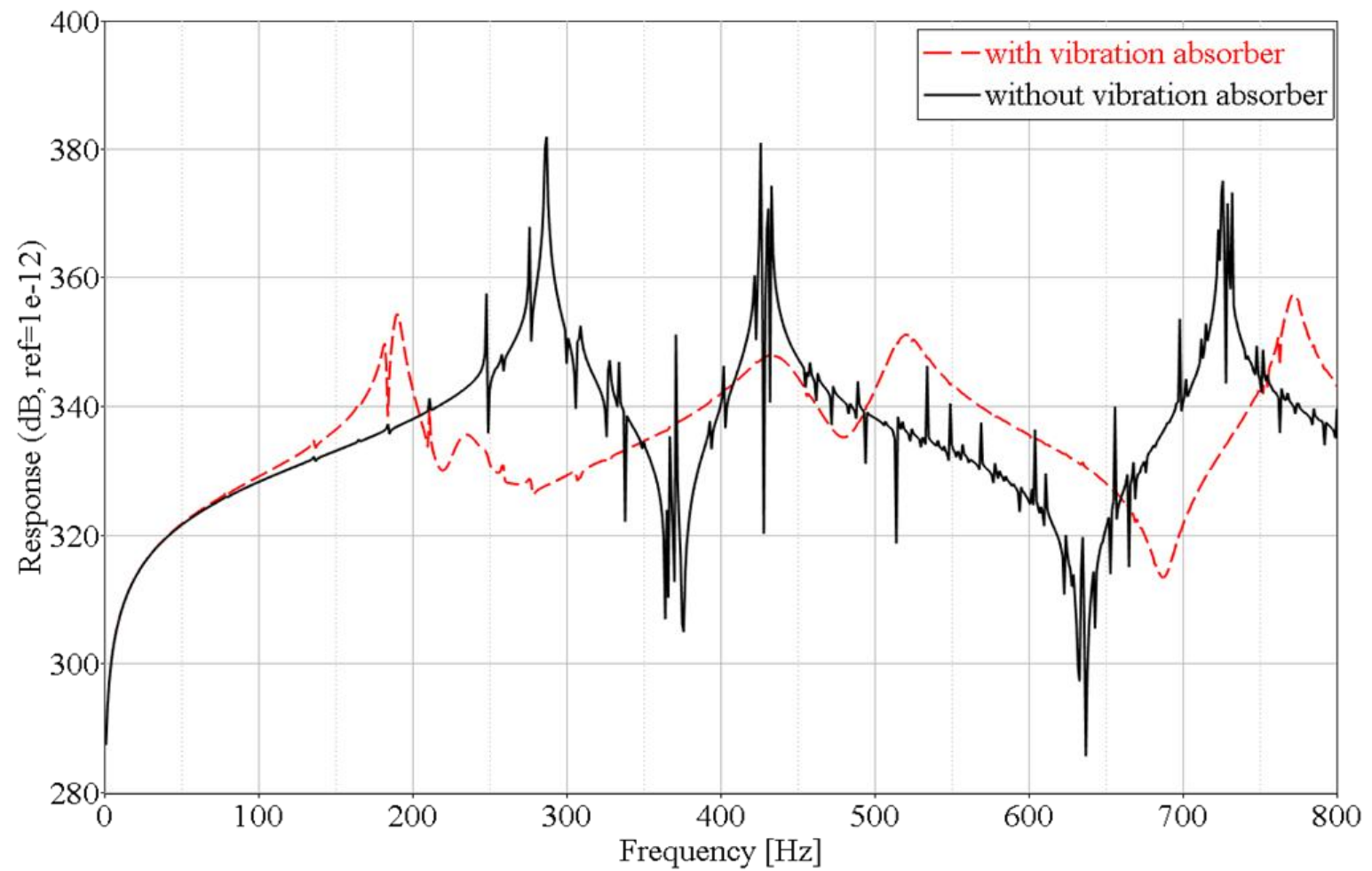

(a)

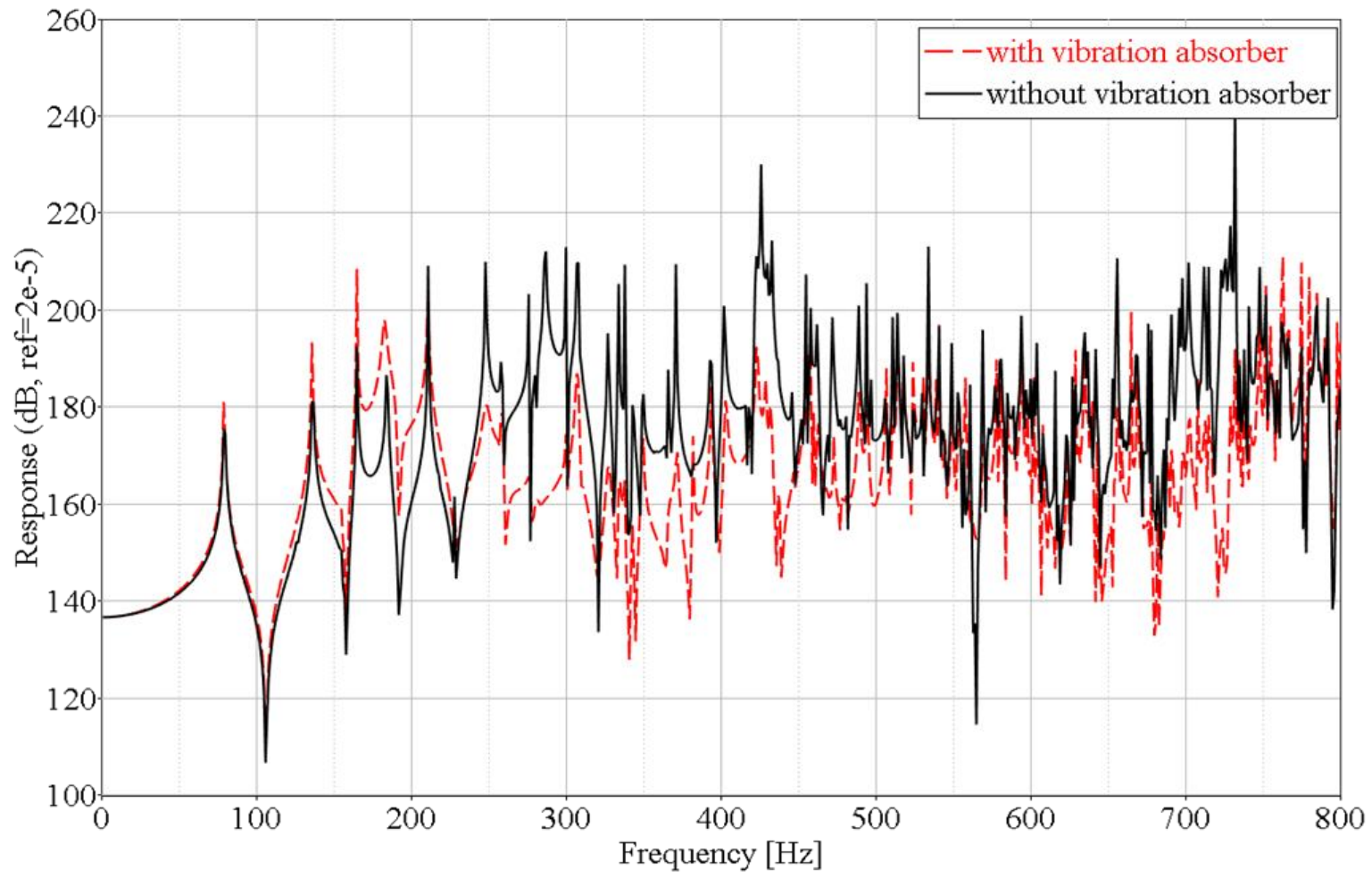

(b)

Fig. 12. The results for analysis of front panel and passenger compartment cavity of vehicle in the structural-acoustic coupled system (a) vibration response at the center of front panel with and without vibration absorbers, and (b) sound pressure level response at driver's ear position with and without vibration absorbers 
